\title{
Quantum Coherent Multielectron Processes in an Atomic Scale Contact
}

Peters, Peter-Jan; Xu, Fei; Kaasbjerg, Kristen; Rastelli, Gianluca; Belzig, Wolfgang; Berndt, Richard

Published in:

Physical Review Letters

Link to article, DOI:

10.1103/PhysRevLett.119.066803

Publication date:

2017

Document Version

Publisher's PDF, also known as Version of record

Link back to DTU Orbit

Citation (APA):

Peters, P-J., Xu, F., Kaasbjerg, K., Rastelli, G., Belzig, W., \& Berndt, R. (2017). Quantum Coherent Multielectron Processes in an Atomic Scale Contact. Physical Review Letters, 119(6), [066803 ].

https://doi.org/10.1103/PhysRevLett.119.066803

\section{General rights}

Copyright and moral rights for the publications made accessible in the public portal are retained by the authors and/or other copyright owners and it is a condition of accessing publications that users recognise and abide by the legal requirements associated with these rights.

- Users may download and print one copy of any publication from the public portal for the purpose of private study or research.

- You may not further distribute the material or use it for any profit-making activity or commercial gain

- You may freely distribute the URL identifying the publication in the public portal 


\title{
Quantum Coherent Multielectron Processes in an Atomic Scale Contact
}

\author{
Peter-Jan Peters, ${ }^{1}$ Fei Xu, ${ }^{2}$ Kristen Kaasbjerg, ${ }^{3}$ Gianluca Rastelli, ${ }^{2}$ Wolfgang Belzig, ${ }^{2}$ and Richard Berndt ${ }^{1, *}$ \\ ${ }^{1}$ Institut für Experimentelle und Angewandte Physik, Christian-Albrechts-Universität zu Kiel, 24098 Kiel, Germany \\ ${ }^{2}$ Fachbereich Physik, Universität Konstanz, 78457 Konstanz, Germany \\ ${ }^{3}$ Center for Nanostructured Graphene, Department of Micro- and Nanotechnology, \\ Technical University of Denmark, 2800 Kongens Lyngby, Denmark
}

(Received 12 April 2017; published 10 August 2017)

\begin{abstract}
The light emission from a scanning tunneling microscope operated on a $\operatorname{Ag}(111)$ surface at $6 \mathrm{~K}$ is analyzed from low conductances to values approaching the conductance quantum. Optical spectra recorded at sample voltages $V$ reveal emission with photon energies $h \nu>2 e V$. A model of electrons interacting coherently via a localized plasmon-polariton mode reproduces the experimental data, in particular, the kinks in the spectra at $\mathrm{eV}$ and $2 \mathrm{eV}$ as well as the scaling of the intensity at low and intermediate conductances.
\end{abstract}

DOI: 10.1103/PhysRevLett.119.066803

A biased nanoscale junction between metal electrodes is a useful environment to study links between quantum transport and electrodynamics. On one hand, very high current densities may be achieved for electrons at energies beyond the Fermi level. On the other hand, such junctions support localized plasmon modes that drastically enhance electromagnetic fields [1-7]. A consequence of this coincidence is that light affects the conductance of the junction [8-10]; another one is the emission of photons, which is driven by the shot noise of the current and corresponds to inelastic tunneling processes [11-14]. Recently, the latter process has been used to electrically drive optical antennas $[15,16]$.

In addition to the emission at energies $h \nu>e V$ (denoted $1 e$ light), where $V$ is the applied bias, higher photon energies $h \nu>e V$ (2e light) have also been observed from metallic and molecular junctions [17-25]. For the observations from single-atom junctions in a scanning tunneling microscope (STM), two models have been proposed. Xu et al. [26,27] considered a single, local plasmon-polariton mode modeled by an $L C$ circuit, and attributed the emission to the nonGaussian quantum noise of the current. Kaasbjerg and Nitzan calculated the current noise to higher order in the electron-plasmon interaction and found that a plasmonmediated electron-electron interaction is the source of experimentally observed above-threshold light emission [28]. The above-mentioned interpretations in terms of coherent processes have been challenged on the basis of experimental data from junctions prepared by electromigration. Heating of the electron gas has been suggested to lead to the emission at $h \nu>e V$ [29]. Blackbody radiation from heated electrons had previously been invoked in Ref. [18].

In this Letter we report on the emission of $1 e, 2 e$, as well as $3 e$ light from junctions between a STM tip and a $\mathrm{Ag}(111)$ single crystal surface. Optical spectra reveal kinks at $h \nu>$ $e V$ and $2 e V$, where $V$ is the sample voltage, and the emission intensity varies in a characteristic manner with the junction conductance. The observations are consistently explained by a model of coherent multielectron scattering off the local plasmon polariton [26-28]. The underlying higherorder processes, sketched in the top panel of Fig. 1 for $3 e$ light, proceed via virtual intermediate states, thereby allowing for the emission of the observed high-energy photons [30].

The model leads to the overall behavior of the emission shown in Fig. 1 (bottom) where processes involving $n=1$,

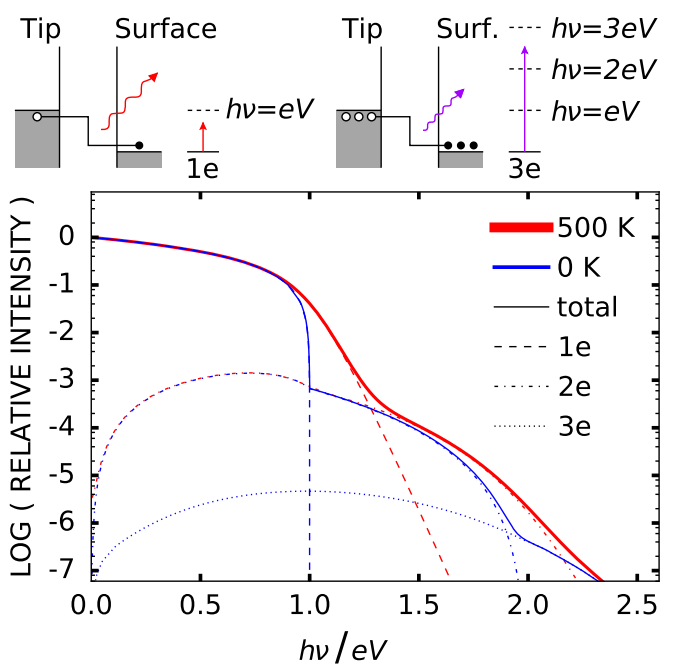

FIG. 1. Top panel: Energy diagrams for single- and multielectron photon emission processes from a STM junction comprising a tip and a surface. In a conventional $1 e$ process (left), tunneling of a single electron leads to the emission of a photon whose energy $h \nu$ (straight arrow) is limited by the voltage $V$ applied to the sample. In a $3 e$ process (right), three electrons interact via a plasmon to generate a photon with $h \nu$ (straight arrow) up to $3 \mathrm{eV}$. Bottom panel: Calculated emission intensity of multielectron processes at $T=0$ and $500 \mathrm{~K}$, normalized to 1 at zero photon energy, vs normalized photon energy $h \nu>e V$. The respective contributions from $1 e, 2 e$, and $3 e$ processes are indicated by dashed, dash-dotted, and dotted lines. A structureless plasmon spectrum and a coupling parameter $\tilde{g}=0.006$ (see text for definition) were used. 
2 and 3 electrons add up to a total intensity that-at low temperature-exhibits characteristic kinks at the photon energies $h \nu=n e V$. Our work identifies the overbias emission and the corresponding kinks as distinct fingerprints of higher-order electron-plasmon scattering processes, and at the same time indicates insignificant electronic heating in STM contacts.

We used an ultrahigh vacuum STM operated at a base temperature of $6 \mathrm{~K} . \mathrm{Ag}(111)$ samples and electrochemically etched Ag tips were prepared by cycles of Ar ion bombardment and annealing. After inserting the tips into the STM, they were processed by repeated contact formation at sample voltages $V$ up to $2 \mathrm{~V}$ until the tips were stable [40]. Experiments were conducted on atomically flat terraces. Light emitted from the tip-sample junction was collected with a lens in vacuo, then focused onto an optical fiber connected to a grating spectrometer and a thermoelectrically cooled CCD camera [41]. The spectrometer/ CCD setup could be exchanged for a photomultiplier tube (PMT) for more sensitive measurement.

Figure 2 shows emission spectra from a $\mathrm{Ag}-\mathrm{Ag}(111)$ junction recorded at low voltages $V=0.9-1.1 \mathrm{~V}$, which were used to keep the photon energy of the more intense $1 e$ light below the detection threshold of $\approx 1 \mathrm{eV}$ [42]. The spectra reveal significant $2 e$ emission, whose intensity drops towards $h \nu=2 e V$ (arrows). However, light at higher energies $h \nu>2 e V$ ( $3 e$ light) is also detected.

For further analysis the spectra $R_{V}$ recorded at a voltage $V$ were normalized by division with a reference spectrum $R_{V_{R}}$ recorded at an elevated bias $V_{R}$ while taking into account the expected linear cutoff of $R_{V_{R}}$ by a weighting factor [1,43,44],

$$
N_{V}(h \nu)=\frac{R_{V}(h \nu)}{R_{V_{R}}(h \nu)}\left(1-\frac{h \nu}{e V_{R}}\right)
$$

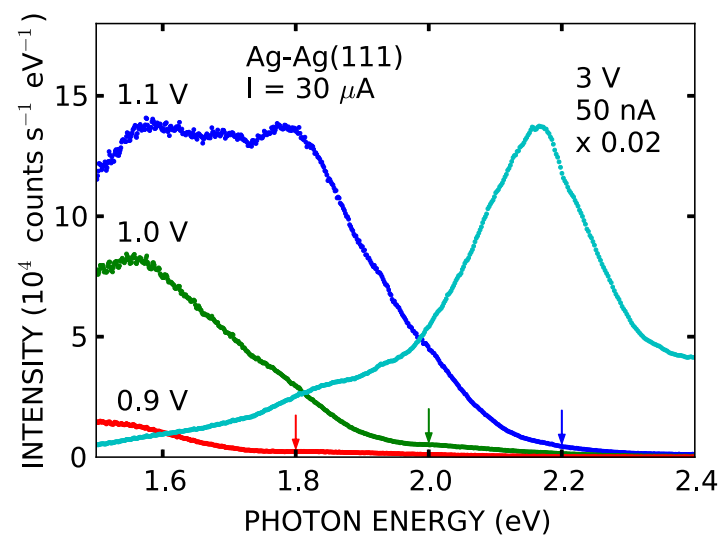

FIG. 2. Spectra of the light emitted from a $\mathrm{Ag}-\mathrm{Ag}(111)$ junction at constant current $I=30 \mu \mathrm{A}$ for three sample voltages. A spectrum recorded at $3 \mathrm{~V}$ and reduced current is also shown, scaled by a factor of 0.02 . Spectra are corrected for dark count rate but not for spectral sensitivity. Arrows indicate thresholds for $3 e$ processes at a photon energy $h \nu=2 e V$.
This procedure removes the effect of the energy-dependent sensitivity of the detection setup [45]. It also reduces the influence of the geometry of the junction on a nm scale [11,46-48]. However, the position and shape of the plasmon resonance depend on the tip-sample distance to some extent [48]. This distance cannot be made identical for measurements at low bias, where $3 e$ emission is discernible, and the higher voltage used to determine the shape of the plasmon resonance. Consequently, as detailed in Supplemental Material [30], the normalization procedure used for Fig. 3(a) does not work well at the onset of the plasmon peak $(h \nu>2 \mathrm{eV}$ ). In Fig. 3(b), however, the voltage of interest $(2.5 \mathrm{~V})$ and the reference voltage $(3.5 \mathrm{~V})$ are rather similar and both spectra could be recorded at identical conductances. The normalization is therefore expected to work throughout the photon energy range shown. We verified that the tip shape did not change during a set of measurements by recording reference spectra and STM images before and after.
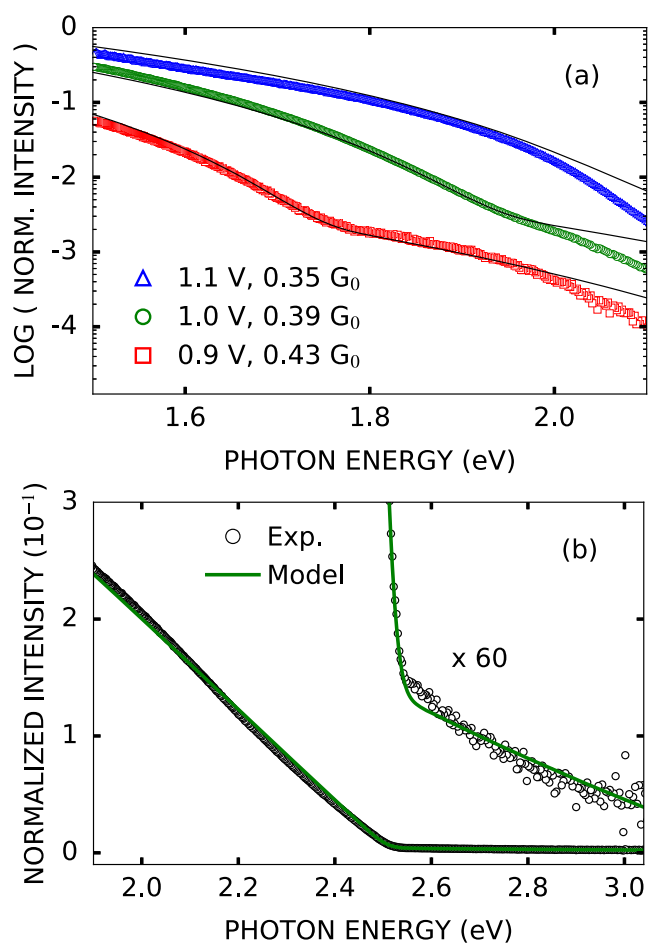

FIG. 3. (a) Spectra from Fig. 2 normalized according to Eq. (1) with the $3 \mathrm{~V}$ data and displayed on a logarithmic scale. Voltages and conductances, in units of $G_{0}=2 e^{2} / h$, are indicated. Lines are fits from the model using the experimental conductances and $\hbar \omega_{0}=2.1 \mathrm{eV}, \eta=0.3 \mathrm{eV}$, a temperature of $6 \mathrm{~K}$ and coupling parameters $\tilde{g}=0.013,0.03$, and 0.05 , respectively. (b) Spectrum of the threshold for $2 e$ light measured at $2.5 \mathrm{~V}$ normalized with $3.5 \mathrm{~V}$ data. The spectrum was recorded at $G=0.1 G_{0}$ with a tip different from (a). The line is a fit from the model assuming a temperature of $55 \mathrm{~K}$, which reproduces the position of the kink as well as its rounding. The other parameters are $\hbar \omega_{0}=2 \mathrm{eV}$, $\eta=0.8 \mathrm{eV}$, and $\tilde{g}=0.011$. Zoomed data have been vertically shifted by 0.1 for clarity. The resolution of the detection setup has been taken into account in panels (a) and (b). 
Figure 3(a) shows normalized data $N_{V}$. The intensity, which is mainly due to $2 e$ light, smoothly drops towards the threshold for $3 e$ light, $h \nu=2 e V$. Importantly, a clear change of slope is observed near the thresholds, which indicates that an additional radiative process becomes relevant. A change of slope is also clearly observed at the transition between the $1 e$ and $2 e$ spectral ranges shown in Fig. 3(b). The observed thresholds are difficult to reconcile with the scenario of Refs. [18] and [29], where the light emission at photon energies $h \nu>e V$ is attributed to spontaneous emission from a hot electron gas. Such a scenario is not consistent with the kinks observed in the emission spectra at $h \nu=n e V$.

To interpret the light emission up to photon energies of $n e V$, we developed a model for higher-order scattering processes between $n$ electrons and the dynamic electric field of a plasmon-polariton resonance by combining the approaches of Refs. [26-28]. The tip-induced plasmon of the STM junction is modeled as a damped $L C$ circuit, which absorbs energy from tunneling electrons and emits photons at energies that may exceed $e V$. The electromagnetic enhancement due to the resonance is given by a Lorentzian $P(E)=\omega_{0}^{2} /\left[\left(\omega_{0}^{2}-E^{2} / \hbar^{2}\right)^{2}+E^{2} \eta^{2}\right]$, where $\omega_{0}=1 / \sqrt{L C}$ is the frequency of the plasmon mode and $\eta$ is a damping parameter. Here $L$ denotes the effective impedance and $C$ the capacitance. The resonance describes both the emission enhancement as well as the effective interaction between the electrons. The coupling coefficient between the current and the plasmon is expressed as $\tilde{g}=G G_{0} L / C$, where $G$ is the conductance and $G_{0}=2 e^{2} / h$.

As detailed in Supplemental Material [30], the rate $R_{1 e}$ of $1 e$ photon emission is governed by the enhancement factor $P$ and $\tilde{S}=S / G$, where $S$ is the shot noise spectral density of the current through noninteracting conductance channels with transmissions $\left\{\tau_{i}\right\}$ [43],

$$
\begin{aligned}
& R_{1 e}(h \nu)=R_{0} \tilde{g} P(h \nu) \tilde{S}(h \nu), \\
S(h \nu)= & G F[w(h \nu-e V)+w(h \nu+e V)] \\
& +2 G(1-F) w(h \nu),
\end{aligned}
$$

with $G=G_{0} \sum_{i} \tau_{i}$, Fano factor $F=\sum_{i} \tau_{i}\left(1-\tau_{i}\right) / \sum_{i} \tau_{i}$, and $w(E)=E n_{B}(E)$ where $n_{B}(E)=\left(e^{E / k_{B} T}-1\right)^{-1}$ is the Bose-Einstein distribution. $R_{0}$ is some reference rate, which includes the detector efficiency and other experimentally unkown parameters.

The rate $R_{2 e}$ of the $2 e$ emission in the frequency range $h \nu>e V$ can at low temperature, $k_{B} T \ll e V, h \nu$, be expressed as

$R_{2 e}(h \nu)=R_{0} \tilde{g}^{2} P(h \nu) \int_{h \nu-e V}^{e V} d \varepsilon P(\varepsilon) \tilde{S}(\varepsilon) \tilde{S}(h \nu-\varepsilon)$,

where $\tilde{S}(\varepsilon)=F(\varepsilon-e V)$ in the integration domain. Correspondingly, the rate $R_{3 e}$ of the $3 e$ emission in the range $h \nu>2 e V$ is

$$
\begin{aligned}
R_{3 e}(h \nu)= & R_{0} \tilde{g}^{3} P(h \nu) \int_{h \nu-2 e V}^{e V} d \varepsilon \int_{h \nu-e V-\varepsilon}^{e V} d \varepsilon^{\prime} \\
& \times P(\varepsilon) \tilde{S}(\varepsilon) \tilde{S}\left(\varepsilon^{\prime}\right) P\left(\varepsilon^{\prime}\right) \tilde{S}\left(h \nu-\varepsilon-\varepsilon^{\prime}\right) .
\end{aligned}
$$

Full expressions for the rates, in particular, at finite temperatures, are provided in Supplemental Material [30].

For illustration, Fig. 1 shows the overall spectra calculated assuming a featureless plasmon resonance, viz. $P(h \nu)=$ const. At low temperature, the intensity rapidly drops at photon energies of $n e V, n=1,2$, as expected for processes involving $n$ electrons. The $1 e$ cutoff is less abrupt and shifts to higher energies at an elevated temperature of $500 \mathrm{~K}$ because of the broadened Fermi distributions of the electrodes. Note that since we only have expressions for the $3 e$ light at $T=0$ and in the $3 e$ regime, we use always the zero temperature expression and extrapolate the curve beyond the $3 e$ regime, but we expect no significant changes in the energy range shown.

Next we use the model to fit experimental spectra. Figure 3(a) shows a comparison of experimental spectra (symbols) recorded at three voltages and the corresponding calculated results (lines) around the $2 e-3 e$ threshold. The model reproduces the spectra fairly well assuming $T \approx 20 \mathrm{~K}$. Deviations mainly occur at $h \nu>2 \mathrm{eV}$, where the normalization of the experimental data is less accurate.

Figure 1 suggests that the $1 e-2 e$ threshold should be more well defined. This is indeed observed in the data [Fig. 3(b), circles] and reproduced by the model spectra (lines). In the model, a temperature of $55 \mathrm{~K}$ was found to lead to an acceptable fit, demonstrating that some heating does occur. A further increase of the temperature would cause a shift of the kink and additional broadening. We conclude that the heating is orders of magnitude lower in our STM junctions than that previously invoked to interpret the emission of $2 e$ and $3 e$ light from electromigrated junctions [29].

A central prediction of our theory [cf., Eqs. (2)-(5)] is that the low-temperature ne emission in the regime $h \nu>(n-1) e V$ scales with the conductance and Fano factor of a general multichannel contact as

$$
R_{n e} \propto G^{n} F^{n} .
$$

This suggests that the Fano factor can be inferred from the ne intensity as $F \sim \sqrt[n]{R_{n e}} / G$. In a simplified scenario of a single conductance channel with transmission $\tau$, conductance $G=G_{0} \tau$, and Fano factor $F=1-\tau$, a scaling $R_{n e} \propto$ $\tau^{n}(1-\tau)^{n}$ previously confirmed for $2 e$ emission [28] is therefore expected.

Figure 4 shows the measured photon intensity in the $3 e$ light regime $h \nu>2 e V$ recorded with two different tips (symbols). The data have been scaled as $R_{3 e}^{1 / 3} / G$ in order to reflect the Fano factor for a single channel in the lowconductance regime, $G \ll G_{0}$. In addition, the Fano factor for a single-channel contact is shown (dashed line). Up to 


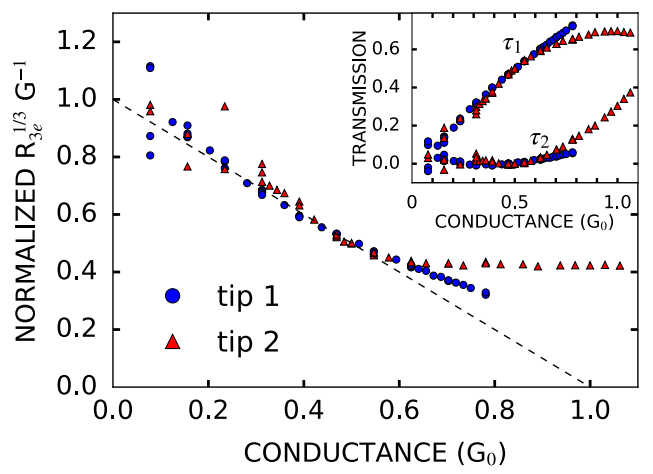

FIG. 4. Intensity $R$ of light vs conductance $G$, scaled as $\sqrt[3]{R} / G$. To reduce the required integration times and thus enable completion of a series of measurements without an unintentional modification of the STM tip the measurements were performed with a PMT. An optical filter was used to limit its sensitivity to photon energies from 1.77 to $3.10 \mathrm{eV}$. Using a sample voltage $V=0.827 \mathrm{~V}$ only photons with energies $h \nu>2 \mathrm{eV}$ were detected. To exclude changes of the tip, approximately the first half of all data were measured at conductances increasing between data points. The second half was recorded at decreasing conductances to fill in the gaps between data points. Circles and triangles show data recorded with different tips. The dashed line indicates the $F=1-G / G_{0}$ behavior expected for a singlechannel contact. The inset shows the transmissions for both tips extracted from a two-channel model with Fano factor (7).

conductance of $\approx 0.5 G_{0}$, Fig. 4 shows a good match of the data and the simplified single-channel expectation. However, at higher conductance $G \gtrsim 0.5 G_{0}$, significant deviations appear. To explain the discrepancy, we consider the opening of additional channels with increasing conductance, which have previously been reported for metal contacts [49-53]. Calculations revealed two almost degenerate channels opening up, which involve states with an angular momentum of $1 \hbar$ around the tip axis [51,54]. In addition, these states presumably are the reason for observed deviations of the $1 e$ yield from a simple $F \sim 1-$ $\tau$ behavior in $\mathrm{Ag}$ and $\mathrm{Cu}$ contacts $[12,55]$.

The model can be made quantitative by assuming that at most two channels with transmissions $\tau_{1}$ and $\tau_{2}$ contribute. For the conductance and Fano factor we then have

$$
G=G_{0}\left(\tau_{1}+\tau_{2}\right) \text { and } F=1-\frac{\tau_{1}^{2}+\tau_{2}^{2}}{\tau_{1}+\tau_{2}}
$$

respectively. Considering $\tau_{1,2}$ to be arbitrary functions of the conductance, we can extract their conductance dependencies from the $3 e$ intensities in Fig. 4 without additional fitting parameters. The result for the variation of the two channel transmissions with the overall conductance is shown in the inset of Fig. 4. The extracted transmission coefficients clearly support the opening of an additional conductance channel at $G \approx 0.5-0.6 \mathrm{G}_{0}$. The intensity for both tips is thus in full accordance with the model invoking two conductance channels without involving any heating. Hence, the light emission from the STM can be explained by coherent plasmon-mediated multielectron processes.

In summary, we have analyzed the emission of $1 e, 2 e$, and $3 e$ light from atomic-scale contacts in a STM. Characteristic features of the spectra, the relative intensities, and the scaling with the conductance are consistently explained in terms of higher-order electron-plasmon interaction. Our results exclude high electron temperatures as being the reason of the overbias light emission in the present STM experiments. Rather, they suggest that it is promising to extend the mesoscopic transport formulation presented here to more complex situations involving, e.g., molecules in the transport path [56-58] or complex interacting plasmon resonances. In antenna structures maximizing the electron-plasmon interaction $[59,60]$ higher-order effects may be further enhanced. Another interesting direction is to consider pulsed bias voltages leading to correlated photon emission.

We thank Tomáš Novotný and Juan-Carlos Cuevas for discussions. K. K. acknowledges support from the European Union's Horizon 2020 research and innovation program under the Marie Sklodowska-Curie Grant No. 713683 (COFUNDfellowsDTU). G. R. acknowledges support by the Excellence Initiative through the Zukunftskolleg. G. R., F. X., and W. B. acknowledge financial support by the Deutsche Forschungsgemeinschaft through SFB 767.

*berndt@physik.uni-kiel.de

[1] J. Lambe and S. L. McCarthy, Phys. Rev. Lett. 37, 923 (1976).

[2] R. Berndt, J. K. Gimzewski, and P. Johansson, Phys. Rev. Lett. 71, 3493 (1993).

[3] T. Atay, J.-H. Song, and A. V. Nurmikko, Nano Lett. 4, 1627 (2004).

[4] I. Romero, J. Aizpurua, G. W. Bryant, and F. J. García De Abajo, Opt. Express 14, 9988 (2006).

[5] K. J. Savage, M. M. Hawkeye, R. Esteban, A. G. Borisov, J. Aizpurua, and J. J. Baumberg, Nature (London) 491, 574 (2012).

[6] D. C. Marinica, M. Zapata, P. Nordlander, A. K. Kazansky, P. M. Echenique, J. Aizpurua, and A. G. Borisov, Sci. Adv. 1, e1501095 (2015).

[7] Y. Vardi, E. Cohen-Hoshen, G. Shalem, and I. Bar-Joseph, Nano Lett. 16, 748 (2016).

[8] R. Möller, U. Albrecht, J. Boneberg, B. Koslowski, P. Leiderer, and K. Dransfeld, J. Vac. Sci. Technol. B 9, 506 (1991).

[9] M. Völcker, W. Krieger, and H. Walther, Phys. Rev. Lett. 66, 1717 (1991).

[10] D. C. Guhr, D. Rettinger, J. Boneberg, A. Erbe, P. Leiderer, and E. Scheer, Phys. Rev. Lett. 99, 086801 (2007).

[11] R. Berndt, J. K. Gimzewski, and P. Johansson, Phys. Rev. Lett. 67, 3796 (1991). 
[12] N. L. Schneider, G. Schull, and R. Berndt, Phys. Rev. Lett. 105, 026601 (2010).

[13] E. Le Moal, S. Marguet, D. Canneson, B. Rogez, E. Boer-Duchemin, G. Dujardin, T. V. Teperik, D.-C. Marinica, and A. G. Borisov, Phys. Rev. B 93, 035418 (2016).

[14] K. Kuhnke, C. Große, P. Merino, and K. Kern, Chem. Rev. 117, 5174 (2017).

[15] P. Bharadwaj, A. Bouhelier, and L. Novotny, Phys. Rev. Lett. 106, 226802 (2011).

[16] J. Kern, R. Kullock, J. C. Prangsma, M. Emmerling, M. Kamp, and B. Hecht, Nat. Photonics 9, 582 (2015).

[17] R. Pechou, R. Coratger, F. Ajustron, and J. Beauvillain, Appl. Phys. Lett. 72, 671 (1998).

[18] A. Downes, P. Dumas, and M. E. Welland, Appl. Phys. Lett. 81, 1252 (2002).

[19] G. Hoffmann, R. Berndt, and P. Johansson, Phys. Rev. Lett. 90, 046803 (2003).

[20] Z.-C. Dong, X.-L. Guo, A. S. Trifonov, P. S. Dorozhkin, K. Miki, K. Kimura, S. Yokoyama, and S. Mashiko, Phys. Rev. Lett. 92, 086801 (2004).

[21] T. Uemura, M. Furumoto, T. Nakano, M. Akai-Kasaya, A. Saito, M. Aono, and Y. Kuwahara, Chem. Phys. Lett. 448, 232 (2007).

[22] G. Schull, N. Néel, P. Johansson, and R. Berndt, Phys. Rev. Lett. 102, 057401 (2009).

[23] Z.-C. Dong, X. L. Zhang, H. Y. Gao, Y. Luo, C. Zhang, L. G. Chen, R. Zhang, X. Tao, Y. Zhang, J. L. Yang, and J. G. Hou, Nat. Photonics 4, 50 (2010).

[24] A. Fujiki, Y. Miyake, Y. Oshikane, M. Akai-Kasaya, A. Saito, and Y. Kuwahara, Nanoscale Res. Lett. 6, 347 (2011).

[25] N. L. Schneider, P. Johansson, and R. Berndt, Phys. Rev. B 87, 045409 (2013).

[26] F. Xu, C. Holmqvist, and W. Belzig, Phys. Rev. Lett. 113, 066801 (2014).

[27] F. Xu, C. Holmqvist, G. Rastelli, and W. Belzig, Phys. Rev. B 94, 245111 (2016).

[28] K. Kaasbjerg and A. Nitzan, Phys. Rev. Lett. 114, 126803 (2015).

[29] M. Buret, A. V. Uskov, J. Dellinger, N. Cazier, M.-M. Mennemanteuil, J. Berthelot, I. V. Smetanin, I. E. Protsenko, G. Colas-des Francs, and A. Bouhelier, Nano Lett. 15, 5811 (2015).

[30] See Supplemental Material at http://link.aps.org/ supplemental/10.1103/PhysRevLett.119.066803 for a more complete presentation of the model and details of the data analysis, which includes Refs. [31-39].

[31] J. Tobiska, J. Danon, I. Snyman, and Y. V. Nazarov, Phys. Rev. Lett. 96, 096801 (2006).

[32] G.-L. Ingold and Y. V. Nazarov, in Single Charge Tunneling (Springer, Boston, 1992), pp. 21-107.

[33] M. H. Devoret, D. Esteve, H. Grabert, G. L. Ingold, H. Pothier, and C. Urbina, Phys. Rev. Lett. 64, 1824 (1990).

[34] M. Kindermann and Y. V. Nazarov, Phys. Rev. Lett. 91, 136802 (2003).

[35] A. Kamenev, Field Theory of Nonequilibrium Systems (Cambridge University Press, Cambridge, 2011).

[36] M. Büttiker, Phys. Rev. B 46, 12485 (1992).

[37] M. Büttiker, A. Prêtre, and H. Thomas, Phys. Rev. Lett. 70, 4114 (1993).
[38] H. Bruus and K. Flensberg, Many-Body Quantum Theory in Condensed Matter Physics (Oxford University Press, Oxford, 2004).

[39] G. B. Lesovik and R. Loosen, JETP Lett. 65, 295 (1997).

[40] A. Castellanos-Gomez, G. Rubio-Bollinger, M. Garnica, S. Barja, A. L. Vázquez de Parga, R. Miranda, and N. Agraït, Ultramicroscopy 122, 1 (2012).

[41] G. Hoffmann, J. Kröger, and R. Berndt, Rev. Sci. Instrum. 73, 305 (2002)

[42] The quantum yields of the various emission components depend on the STM tip, the conductance, and the voltage. We recorded data for $1 e, 2 e$, and $3 e$ light at $V=3.0,1.1$, and $0.8 \mathrm{~V}$, respectively, with the same tip. Assuming isotropic emission the estimated quantum yield of $1 e$ light was $10^{-5}$ at $G=0.02 G_{0}$ with this tip. The yields for $2 e$ and $3 e$ light at $G \approx 0.4 G_{0}$ were lower by factors of $\approx 300$ and $\approx 300^{2}$, respectively.

[43] R. Aguado and L. P. Kouwenhoven, Phys. Rev. Lett. 84, 1986 (2000).

[44] Y. M. Blanter and M. Büttiker, Phys. Rep. 336, 1 (2000).

[45] N. L. Schneider, F. Matino, G. Schull, S. Gabutti, M. Mayor, and R. Berndt, Phys. Rev. B 84, 153403 (2011).

[46] R. W. Rendell and D. J. Scalapino, Phys. Rev. B 24, 3276 (1981).

[47] P. Johansson, R. Monreal, and P. Apell, Phys. Rev. B 42, 9210 (1990).

[48] J. Aizpurua, G. Hoffmann, S. P. Apell, and R. Berndt, Phys. Rev. Lett. 89, 156803 (2002).

[49] E. Scheer, N. Agraït, J. C. Cuevas, A. L. Yeyati, B. Ludoph, A. Martín-Rodero, G. R. Bollinger, J. M. van Ruitenbeek, and C. Urbina, Nature (London) 394, 154 (1998).

[50] E. Scheer, W. Belzig, Y. Naveh, M. H. Devoret, D. Esteve, and C. Urbina, Phys. Rev. Lett. 86, 284 (2001).

[51] J.-T. Lü, R. B. Christensen, and M. Brandbyge, Phys. Rev. B 88, 045413 (2013).

[52] R. Vardimon, M. Klionsky, and O. Tal, Phys. Rev. B 88, 161404 (2013).

[53] M. Polok, D. V. Fedorov, A. Bagrets, P. Zahn, and I. Mertig, Phys. Rev. B 83, 245426 (2011).

[54] M. Brandbyge (private communication).

[55] A. Burtzlaff, N. L. Schneider, A. Weismann, and R. Berndt, Surf. Sci. 643, 10 (2016).

[56] N. L. Schneider, J. T. Lü, M. Brandbyge, and R. Berndt, Phys. Rev. Lett. 109, 186601 (2012).

[57] G. Reecht, F. Scheurer, V. Speisser, Y. J. Dappe, F. Mathevet, and G. Schull, Phys. Rev. Lett. 112, 047403 (2014).

[58] B. Doppagne, M. C. Chong, E. Lorchat, S. Berciaud, M. Romeo, H. Bulou, A. Boeglin, F. Scheurer, and G. Schull, Phys. Rev. Lett. 118, 127401 (2017).

[59] M. Parzefall, P. Bharadwaj, A. Jain, T. Taniguchi, K. Watanabe, and L. Novotny, Nat. Nanotechnol. 10, 1058 (2015).

[60] F. Bigourdan, J.-P. Hugonin, F. Marquier, C. Sauvan, and J.-J. Greffet, Phys. Rev. Lett. 116, 106803 (2016). 